\title{
Mono and Bis-Triazoles Incorporated into Chimeric AGRP- Melanocortin Peptide Template as Disulfide-Bridge Mimetics
}

\author{
Srinivasa R. Tala, Anamika Singh, Sathya M. Schnell, Katie Freeman, \\ and Carrie Haskell-Luevano \\ Department of Medicinal Chemistry, University of Minnesota, Minneapolis, MN, 55455, USA
}

\section{Introduction}

The melanocortin receptor (MCR) family consists of five receptor subtypes (MC1R-MC5R), endogenous agonists and antagonists. These receptors are involved in important biological pathways such as skin and hair pigmentation, food intake, energy homeostasis, erectile function, and exocrine glands function [1]. The MC3 and MC4 receptors are expressed in the brain and regulate weight and energy homeostasis as well as feeding behavior [2]. Our laboratory is involved in the design, synthesis and characterization of ligands to probe selectivity and potency at these functionally overlapping receptors [3-5]. The [1,2,3]-triazole-based bridge was previously utilized as a disulfide-bridge replacement in the sunflower trypsin inhibitor-1 peptide [6]. More recently, [1,2,3]-triazole-bridge was introduced into a synthetic melanocortin agonist, MT-II template as a type-I $\beta$-turn mimetic [7]. In our ongoing efforts to identify potent and selective lead compounds at melanocortin receptors, we designed and synthesized mono- and bis-triazoles incorporated chimeric AGRP-Melanocortin peptides. It was hypothesized that triazole-bridge may serve as a viable repacement for the disulfide-bridge in the AGRP-melanocortin chimeric peptide template.

\section{Results and Discussion}

This study utilized the chimeric peptide template Tyr-c[Cys-His-DPhe-Arg-Trp-Asn-Ala-Phe-Cys]Tyr- $\mathrm{NH}_{2}$ (AMW3-130), which we previously identified as a subnanomolar potent agonist for the melanocortin receptors [4,5]. This template is derived from the antagonist hAGRP(109-118) decapeptide sequence with amino acid residues His-Phe-Arg-Trp from the melanocortin agonists incorporated in the place of the Arg-Phe-Phe amino acids. It contains a single disulfide bridge between the two Cys side chains [4,5]. The cyclic peptide AMW3-130 (Table 1) is equipotent to $\alpha-M S H$ at the mMC1R and more potent than $\alpha$-MSH at the mMC4R. Here, we designed 1,4-disubstituted and 1,5disubstituted mono-[1,2,3]-triazole-bridged cyclic peptides $\mathbf{1}$, and bis-[1,2,3]-triazole-bridged cyclic peptides 2 (Figure 1). Linear peptides were synthesized using microwave-assisted Fmoc-solid phase peptide methodology. Copper(I)-catalyzed intramolecular azide-alkyne cycloaddition was used to afford 1,4-disubstituted [1,2,3]-triazole-bridged cyclic peptides. Ruthenium(II)-catalyzed azide-alkyne cycloaddition was used to obtain 1,5-disubstituted [1,2,3]-triazole-bridged cyclic peptides. The

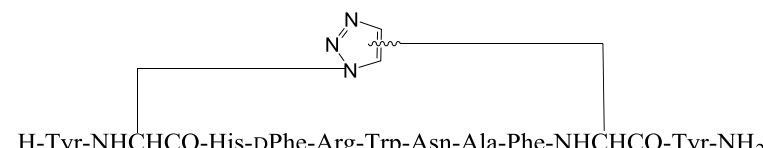

H-Tyr-NHCHCO-His-DPhe-Arg-Trp-Asn-Ala-Phe-NHCHCO-Tyr-NH

1

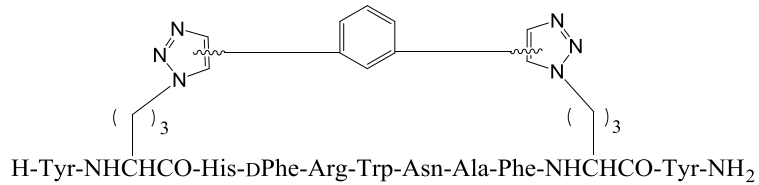

2

Fig. 1. The mono-[1,2,3]- and bis-[1,2,3]-triazolebridged cyclic peptides. purified peptides were at least $95 \%$ pure as determined by RP-HPLC in two diverse solvent systems and possessed the correct molecular weights as determined by mass spectrometry. The synthesized peptides were tested for agonist pharmacology using the cAMP-based AlphaScreen assay at the mouse melanocortin receptors (mMC3R and mMC4R) [8]. This study resulted in several compounds with interesting pharmacology (Table 1; Figure 2) at mMC3R and mMC4R. The 1,5-disubstituted [1,2,3]-triazolebridged peptide SRT4-79-1cy showed increased agonist activity at the mMC3R and mMC4R compared to the linear compound SRT4-79-2-lin having the azido and alkynyl side chains. 
The 1,5-disubstituted triazolyl peptide SRT479-1cy resulted in equipotent functional activity (within experimental error) at the mMC3R and 5-fold reduced potency at the mMC4R versus the disulfide bridged peptide AMW3-130 (Table 1). The 1,5-disubstituted triazolyl peptide (SRT4-79-1cy) was 4-fold more potent at the $\mathrm{mMC} 3 \mathrm{R}$, but retained equipotency at the mMC4R in comparison to the 1,4-disubstituted triazolyl peptide (SRT479-2cy). The bis-[1,2,3]-triazole-bridged peptides SRT4-154-1cy (1,5-disubstituted) and SRT4-154-2cy (1,4-disubstituted) (Figure 1) were designed to improve selectivity at the melanocortin receptors. However, incorporation of the bulky group resulted in reduced agonist potency at both mMC3 and mMC4 receptors (Table 1, Figure 2).

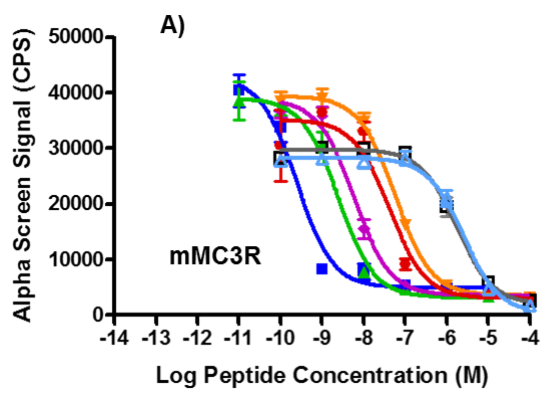

Table 1. Pharmacology of the peptides at the mouse MC3 and MC4 Receptors.

\begin{tabular}{ccc}
\hline \multirow{2}{*}{ Compound } & \multicolumn{2}{c}{ Agonist Potency $(\mathrm{nM})$} \\
\cline { 2 - 3 } & $m M C 3 R$ & $m M C 4 R$ \\
\hline -MSH & 0.15 & 2.7 \\
AMW3-130 & 2.2 & 0.43 \\
SRT4-79-2-lin & 51 & 29 \\
SRT4-79-1cy & 6.4 & 2.0 \\
SRT4-79-2cy & 28 & 6.0 \\
SRT4-154-1cy & 1944 & 971 \\
SRT4-154-2cy & 2744 & 539 \\
\hline
\end{tabular}

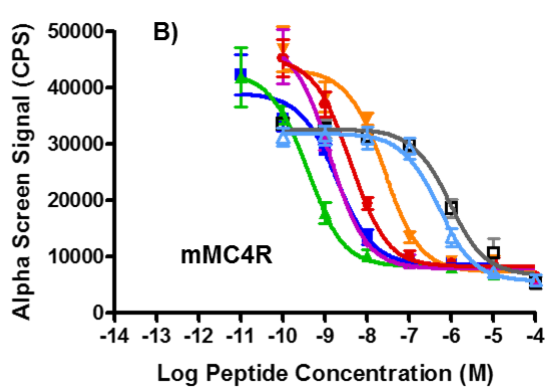

Fig. 2. Illustration of the pharmacological agonist dose-response curve for the synthesized peptides characterized in this study at the A) $m M C 3 R$ and B) $m M C 4 R$.

In conclusion, we synthesized 1,4- and 1,5-disubstituted triazole-bridged macrocyclic peptides using $\mathrm{Cu}(\mathrm{I})$-catalyzed and $\mathrm{Ru}(\mathrm{II})$-catalyzed cycloadditions respectively. We demonstrated that the 1,2,3triazole-bridge is a viable mimetic for disulfide-bridge replacement in melanocortin chimeric peptide template. These triazole-bridged peptides may serve as a probe for in vivo studies.

\section{Acknowledgments}

This study was supported by NIH Grant NIHRO1DK091906.

\section{References}

1. Irani, B.J., et al. Curr. Pharma. Design 10, 3443-3479 (2004), http://dx.doi.org/10.2174/1381612043382891

2. Huszar, D., et al. Cell 88, 131-141 (1997), http://dx.doi.org/10.1016/S0092-8674(00)81865-6

3. Xiang, Z., et al. Biochemistry 46, 8273-8287 (2007), http://dx.doi.org/10.1021/bi7007382

4. Wilczynski, A., et al. J. Med. Chem. 47, 2194-2207 (2004), http://dx.doi.org/10.1021/jm0303608

5. Singh, A., et al. J. Med. Chem. 54, 1379-1390 (2011), http://dx.doi.org/10.1021/jm101425m

6. Empting, M., et al. Angew. Chem. Int. Ed. 50, 5207-5211 (2011), http://dx.doi.org/10.1002/anie.201008142

7. Testa, C., et al. J. Med. Chem. 57, 9424 (2014), http://dx.doi.org/10.1021/jm501027w

8. Singh, A., et al. ACS Med. Chem. Lett. 6, 568-578 (2015), http://dx.doi.org/10.1021/acsmedchemlett.5b00053 